\title{
Negative pressure wound treatment improves Acute Physiology and Chronic Health Evaluation II score in mediastinitis allowing a successful elective pectoralis muscle flap closure: Six-year experience of a single protocol
}

\author{
Andrea Salica, MD, Luca Weltert, MD, Raffaele Scaffa, MD, Lorenzo Guerrieri Wolf, MD, \\ Saverio Nardella, MD, Alessandro Bellisario, MD, and Ruggero De Paulis, MD
}

\begin{abstract}
Objectives: Optimal management of poststernotomy mediastinitis is controversial. Negative pressure wound treatment improves wound environment and sternal stability with low surgical invasiveness. Our protocol was based on negative pressure followed by delayed surgical closure. The aim of this study was to provide the results at early follow-up and to identify the risk factors for adverse outcome.

Methods: In 5400 cardiac procedures, 44 consecutive patients with mediastinitis were enrolled in the study. Mediastinitis treatment was based on urgent debridement and negative pressure as the first-line approach. After wound sterilization, chest closure was achieved by elective pectoralis muscle advancement flap. Each patient's hospital data were collected prospectively. Variables included patient demographics and clinical and biological data. Acute Physiology and Chronic Health Evaluation (APACHE) II score was calculated at the time of diagnosis and 48 hours after debridement. Focus outcome measures were mediastinitis-related death and need for reintervention after pectoralis muscle closure.
\end{abstract}

Results: El Oakley type I and type IIIA mediastinitis were the most frequent types (63.6\%). Methicillinresistant Staphylococcus aureus was present in 25 patients (56.8\%). Mean APACHE II score was $19.4 \pm 4$ at the time of diagnosis, and 30 patients $(68.2 \%)$ required intensive care unit transfer before surgical debridement. APACHE II score improved 48 hours after wound debridement and negative pressure application (mean value, $19.4 \pm 4$ vs $7.2 \pm 2 ; P=.005)$ independently of any other variables included in the study. One patient in septic shock at the time of diagnosis died $(2.2 \%)$.

Conclusions: Negative pressure promotes a significant improvement in clinical status according to APACHE II score and allows a successful elective surgical closure. (J Thorac Cardiovasc Surg 2014;148:2397-403)

Poststernotomy mediastinitis (PSM) is a rare but lifethreatening complication with an incidence of $1 \%$ to $3 \%$ of cardiac procedures. ${ }^{1,2}$ PSM is one of the most expensive complications in cardiac surgery with a 3 -fold increase in overall costs. ${ }^{3}$ Optimal management is still controversial. The mortality rate remains high, ranging from $5 \%$ to $47 \%,{ }^{4-6}$ with a recurrence of approximately $20 \%$.

The open wound approach allows infection control by serial wound dressing changes, but the high mortality rate, up to $45 \%$, induced many centers to abandon this strategy. ${ }^{8}$ Closed techniques are based on an aggressive surgical approach. Extensive debridement and primary chest wall irrigation or suction closure are widely accepted and

\footnotetext{
From the Department of Cardiac Surgery, European Hospital, Rome, Italy. Disclosures: Authors have nothing to disclose with regard to commercial support. Received for publication Feb 18, 2014; revisions received March 31, 2014; accepted for publication April 11, 2014; available ahead of print May 14, 2014. Address for reprints: Ruggero De Paulis, MD, Division of Cardiac Surgery, European Hospital, via Portuense 700, 00149 Rome, Italy (E-mail: depauli@tin.it). $0022-5223 / \$ 36.00$

Copyright (c) 2014 by The American Association for Thoracic Surgery http://dx.doi.org/10.1016/j.jtcvs.2014.04.025
}

achieve immediate chest wall integrity, although wound closure and infection healing do not coincide.

The introduction of vacuum-assisted closure $(\mathrm{VAC})^{9,10}$ changed the management of sternal wound complications toward a less-invasive approach. Negative pressure improves the wound environment and restores sternal stability with minimal surgical stress and a shorter intensive care unit (ICU) stay than primary closed or open packing treatments. ${ }^{11,12}$

Despite several efforts and a multidisciplinary approach, the outcome of deep sternal wound infections is often unpredictable. Mediastinitis is a high-risk condition in which patient baseline characteristics are worsened by infection, sternal instability, and recent cardiac surgery. Mediastinitis mortality risk factors, such as age, renal failure, obesity, severity of illness, persistently positive bacteremia, and prolonged mechanical ventilation after wound debridement, highlight the frailty of this population. ${ }^{12-14}$

Over a 6-year period, we have adopted a single protocol in the treatment of PSM. Our strategy is based on wound debridement and negative pressure wound treatment (NPWT) as first-line treatment until wound sterilization, 


\section{Abbreviations and Acronyms \\ APACHE $=$ Acute Physiology and Chronic Health Evaluation \\ $\mathrm{CABG}=$ coronary artery bypass grafting \\ ICU = intensive care unit \\ MRSA = methicillin-resistant Staphylococcus aureus \\ NPWT = negative pressure wound treatment \\ PMV = prolonged mechanical ventilation \\ PSM = poststernotomy mediastinitis \\ VAC = vacuum-assisted closure}

followed by delayed sternal rewiring and pectoralis muscle flap closure.

We choose NPWT because it minimizes surgical invasiveness. Negative pressure restores sternal stabilization without the need for adjunctive procedures. Continuous drainage improves the wound environment and reduces the bacterial count, allowing a less-invasive approach at the time of surgical debridement. The aim of this study is to provide the results of our single protocol at early follow-up and to identify the risk factors for an adverse outcome.

\section{METHODS}

This observational study was conducted in a single cardiac center. Between April 2006 and September 2013 at the European Hospital of Rome, 5400 midline sternotomies for cardiac procedures were performed.

Patients with deep sternal dehiscence were assessed in terms of general signs and symptoms. Fever, sternal instability, and blood tests were first evaluated. Samples for microbiological investigation were collected from wound exudation. Computed tomography scan was used to evaluate the presence of mediastinal or pleural fluid collection.

PSM was defined according to the guidelines of the US Centers for Disease Control and Prevention. ${ }^{15}$ Mediastinitis was then classified according to El Oakley and Wright ${ }^{16}$ classification. The Acute Physiology and Chronic Health Evaluation (APACHE) II score ${ }^{17}$ was assessed to evaluate the severity of clinical status before surgical debridement.

Patients with other wound complications, such as superficial wound infections, fat necrosis, and uninfected sternal instability, were excluded from the study. According to this definition, PSM developed in 44 patients $(0.81 \%)$ during the study period, and they were enrolled in the study.

\section{Mediastinitis Management}

Intravenous vancomycin $500 \mathrm{mg}$ every 12 hours was started until the antibiotic regimen was changed according to culture sensitivity and infectologist evaluation. Patients were admitted to the operating room as urgent cases. Sternal wounds were completely reopened. All sternal wires, bone samples, sutures, and necrotic and infected tissues were gently removed and cultured without excessive tissue removal. Procedures were carried out in a conservative manner with minimal removal of bone tissue. The entire field was largely irrigated with hydrogen peroxide, saline solution, and diluted povidone-iodine. When vascular Dacron prostheses were exposed in the field ( 2 cases) the great omentum was prepared and fixed to cover the vascular graft. Hemostasis was accurately completed with diathermy. A sterile polyurethane foam dressing with an open-pore structure was tailored to fit in the wound. A first layer was placed in the sternum and tailored to completely fit the whole dead space between the 2 sternal edges (Figure 1). A second layer, placed over the first, was tailored to fit the whole area between subcutaneous tissues. Both sponges were significantly oversized to completely obliterate dead space due to dehiscence after negative pressure was started. This allows an effective sternal stabilization that promotes respiratory function and prevents organ injury, minimizing sternal movement during patients' mobilization. If needed, 4 layers of paraffin gauzes were placed to protect underlying structures. The wound was sealed with transparent adhesive drape, and the sponge was connected to a pump unit with a continuous negative pressure of $-125 \mathrm{~mm} \mathrm{Hg}$.

Clinical reassessment was performed immediately after debridement and daily for the first 3 postoperative days. Subsequent routine examinations were performed as needed.

NPWT dressing was changed every 2 days or whenever drainage exceeded $200 \mathrm{~mL} / 24$ hours in the surgical ward without the need for anesthetics. Wound samples were cultured each time, and iodine solution was used for dehiscence washing. Full patient mobilization and normal dietary regimen were achieved as soon as possible. After 3 consecutive negative mediastinal cultures, patients were readmitted to the operating room.

Under general anesthesia, sternal rewiring and bilateral pectoralis muscle flap were performed. Fibrous tissue on the wound edge was removed. Bilateral pectoralis muscle flap was performed, raising the inner face of the pectoralis from the sternal and costal insertion to the anterior axillary line. Superiorly, the dissection stopped at the level of the clavicle. The pectoralis minor and the humeral insertion of the pectoralis major were left intact. To minimize closure tensions, a small flap of subcutaneous tissue was made over the pectoralis plane. Sternal rewiring was achieved with 6 to 8 simple interrupted stainless-steel wires. Muscles were advanced to the midline and closed with absorbable interrupted suture (Figure 2). One drainage was put under each muscle flap.

\section{Data Collection}

All patients' hospital data were collected prospectively in our institutional database for surgical wound complications at each patient assessment. Data recorded at the time of wound diagnosis and 48 hours after debridement were analyzed in the study and are listed in Table 1. Diabetes was defined as the need for antidiabetic drugs; obesity was defined as body mass index greater than $30 \mathrm{~kg} / \mathrm{m}^{2}$; anemia was defined as hemoglobin level less than $10 \mathrm{~g} / \mathrm{dL}$ before debridement; chronic obstructive pulmonary disease was defined as long-term use of bronchodilators or steroids; arteriopathy was determined by the presence of stenosis greater than $50 \%$ on the limb or carotid arteries; the type of mediastinitis was defined according to El Oakley and Wright ${ }^{16}$ classification; the time interval to mediastinitis presentation was defined as the interval between the initial cardiac surgery and the mediastinitis diagnosis; and the severity of illness was stratified according to the APACHE II score. The microorganism responsible for mediastinal infection was defined by the cultures of the mediastinal samples, and the presence of concomitant bloodstream infection was evaluated by the results of the blood cultures before wound debridement. Other data were recorded 48 hours after debridement: (1) APACHE II score value, (2) persistence of positive wound cultures, and (3) persistently positive bacteremia, defined as 2 or more positive blood cultures drawn more than 24 hours after debridement (Table 1).

Primary outcome measures were in-hospital death from any cause, mediastinitis-related death (defined as death occurring as a consequence of uncontrollable sepsis, massive mediastinal hemorrhage, or secondary prosthetic infection), and need for reintervention (defined as need for redebridement after pectoralis flap wound closure because of continued local infection or recurrence of sternal instability).

Secondary outcome measures included the following: life-threatening complications, defined as secondary endocarditis, massive mediastinal hemorrhage occurring after debridement, and occurrence of sepsis or multiple-organ failure; ICU stay, defined as need for ICU surveillance 

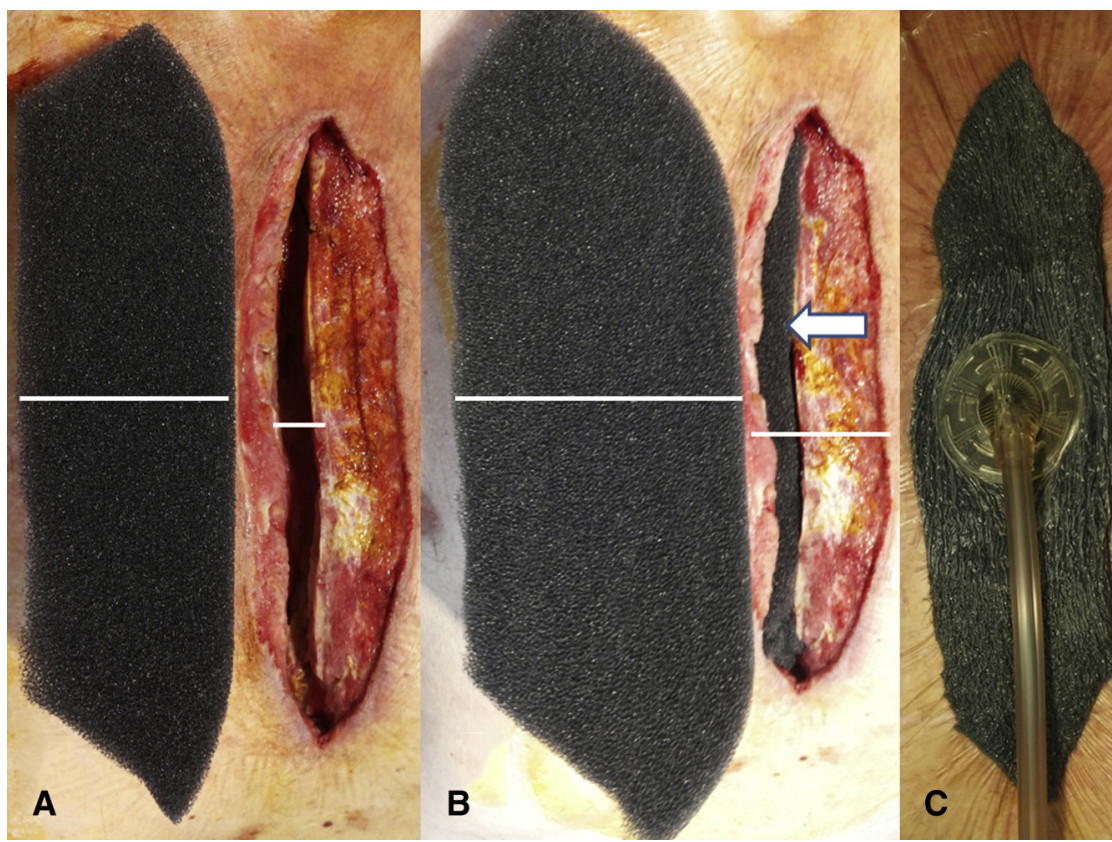

FIGURE 1. A, The first layer of sterile polyurethane foam dressing was tailored to largely oversize the dead space between the sternal edges as shown by the white lines. B, The first layer of the sponge is placed between the sternal edges as shown by the arrow. A second layer was oversized to completely fit the dead space between the presternal tissues (white lines). C, When negative pressure was applied, the whole dead space of the dehiscence was completely obliterated.

more than 24 hours after wound debridement; prolonged mechanical ventilation, defined as need for mechanical ventilation more than 24 hours after debridement; length of stay, calculated after the diagnosis of mediastinitis even if it occurred during the same hospitalization as the initial cardiac surgery; and time required to sterilize mediastinal samples, calculated as the interval between the debridement and the first of the 3 consecutive sterile mediastinal cultures. The European Hospital Institutional Review Board approved the study design.

\section{Statistical Analysis}

All data analysis was carried out with SPSS 20.0 (IBM Corp, New York, NY). Data collection was performed prospectively with Excel datasheets (Microsoft Corp, Redmond, Wash). All binary parameters were standardized to 0 (nonpresence) and 1 (presence) of condition. Contingency tables were obtained for each dependent parameter in relation to the following outcome measures: mediastinitis-related death and need for reintervention. On each table, Pearson K-square test with correction for

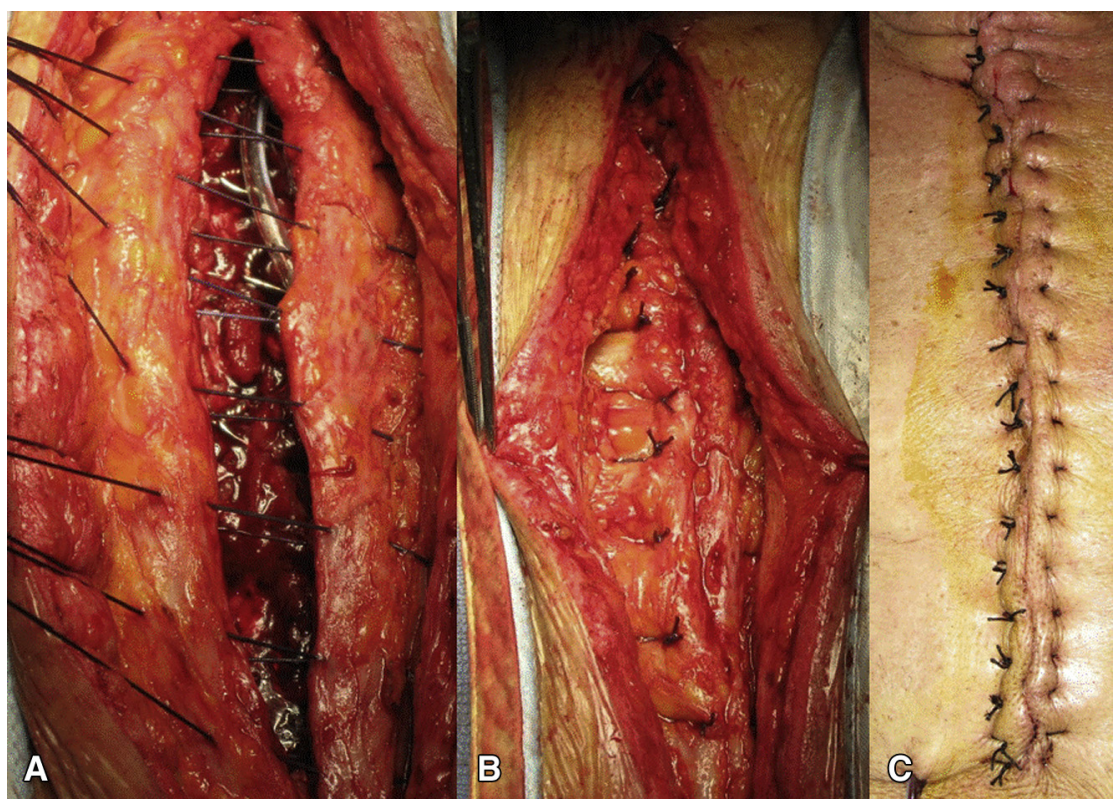

FIGURE 2. A, Sternal rewiring with interrupted stainless-steel wires and Redon drainage under the muscle flap. B, The pectoralis major flaps are advanced to the midline and closed with absorbable interrupted suture. C, Final view of the wound sutured with Donati stitches. 
TABLE 1. Demographic and clinical characteristics of patients at the time of mediastinitis diagnosis and at $\mathbf{4 8}$ hours after debridement and negative pressure wound treatment application

\begin{tabular}{lc}
\multicolumn{1}{c}{ Variables } \\
\hline General characteristics \\
Age, mean \pm SD, $\mathrm{y}$ & $68.4 \pm 10$ \\
Male, $\mathrm{n}(\%)$ & $31(70.4)$ \\
Diabetes, $\mathrm{n}(\%)$ & $33(75)$ \\
Obesity, $\mathrm{n}(\%)$ & $11(25)$ \\
COPD, $\mathrm{n}(\%)$ & $20(45.4)$ \\
Chronic dialysis, $\mathrm{n}(\%)$ & $3(6.8)$ \\
CABG alone, $\mathrm{n}(\%)$ & $25(56.8)$ \\
BITA, $\mathrm{n}(\%)$ & $27(61.3)$ \\
At the time of mediastinitis diagnosis & \\
Leukocyte count (mean value, $\left.\times 10^{3} / \mathrm{mm}^{3}\right)$ & $18.8 \pm 6$ \\
Anemia, $\mathrm{n}(\%)$ & $38(86.36)$ \\
Time interval to mediastinitis presentation, $\mathrm{d}$, & $18.91 \pm 6$ \\
mean \pm SD & \\
Type of mediastinitis (El Oakley) & \\
I & \\
IIIA & $4(9.09 \%)$ \\
IIIB & $24(54.54 \%)$ \\
IVA & $12(27.27 \%)$ \\
APACHE II score, $\mathrm{n}$ & $4(9.09 \%)$ \\
Need for ultrafiltration (new onset), $\mathrm{n}(\%)$ & $19.4 \pm 4$ \\
MRSA in mediastinal cultures, $\mathrm{n}(\%)$ & $4(9)$ \\
Bloodstream infection, $\mathrm{n}(\%)$ & $25(56.8)$ \\
Need for vasoconstrictors, $\mathrm{n}(\%)$ & $27(61.3)$ \\
Septic shock & $15(29.3)$ \\
ICU transfer & $1(2.2 \%)$ \\
48 h after debridement & $30(68.2 \%)$ \\
APACHE II score, mean \pm SD & \\
Positive mediastinal cultures, $(\%)$ & $7.2 \pm 2$ \\
Persistently positive bacteremia, $\mathrm{n}(\%)$ & $44(100)$ \\
\hline APACHE, Acy & $22(50)$ \\
\hline
\end{tabular}

APACHE, Acute Physiology and Chronic Health Evaluation; BITA, bilateral internal thoracic artery; $C A B G$, coronary artery bypass grafting; $C O P D$, chronic obstructive pulmonary disease; ICU, intensive care unit; MRSA, methicillin-resistant Staphylococcus aureus; $S D$, standard deviation.

continuity equation was applied to determine significance. Parametric tests (Wilcoxon and Student $t$ tests for paired samples) were used to verify differences in continuous variables. Preoperative and postprocedural ( 48 hours after debridement) APACHE II score value, results of wound cultures, and results of blood cultures were compared by using the McNemar test. Afterward, univariate analysis was performed with a binary logistic regression equation, with $95 \%$ confidence interval.

\section{RESULTS}

\section{Patients' Characteristics}

The population comprised 31 male and 13 female patients. The mean patient age was $68.4 \pm 10.3$ years. The baseline characteristics are shown in Table 1. Prior cardiac operation was isolated coronary artery bypass grafting $(\mathrm{CABG})$ in 25 patients $(56.8 \%)$; valve procedures or ascending aortic surgery in 9 patients $(20.4 \%)$ (re-do surgery in 2); and CABG associated with valve procedures in 10 patients $(22.7 \%)$. Preoperative risk factors for mediastinitis included obesity (12 patients, 25\%), diabetes
(75.14\%), and chronic obstructive pulmonary disease $(45.4 \%)$. The bilateral internal thoracic artery was harvested in $61.3 \%$ of patients with mediastinitis and was always associated with 1 or more of the following comorbidities: obesity, diabetes, or chronic obstructive pulmonary disease. Renal insufficiency requiring chronic dialysis was present in 3 patients with mediastinitis.

\section{Mediastinitis}

The spectrum of encountered organisms was similar to that in other reports. ${ }^{11,13,14}$ Methicillin-resistant Staphylococcus aureus (MRSA) was identified in 25 patients $(56.8 \%)$, methicillin-susceptible $S$. aureus was identified in 4 patients, and coagulase-negative staphylococci were identified in 4 patients. Gram-negative organisms were identified in 11 patients $(6$ were positive for Pseudomonas aeruginosa and 5 were positive for Escherichia coli).

The mean time interval to mediastinitis presentation was $18.91 \pm 6$ days. El Oakley type I and IIIA were identified in 28 patients $(63.6 \%)$. Mean APACHE II score was $19.4 \pm 4$. Thirty patients $(68.2 \%)$ required ICU transfer before surgical debridement. Four patients $(9.09 \%)$ had acute renal failure and required ultrafiltration. Concomitant bloodstream infection was identified in 27 patients $(61 \%)$. Fifteen patients had systemic signs of sepsis with the need for intravenous vasoconstrictors, 1 of whom had septic shock and required mechanical ventilation before debridement.

Data recorded 48 hours after debridement showed that the mean APACHE II score was significantly improved compared with the preoperative value $(19.4 \pm 4$ vs $7.2 \pm$ $2, P<.005)$, whereas bloodstream infection and mediastinal cultures did not show any differences.

\section{Outcome}

The overall in-hospital mortality rate was $2.2 \%$ (1/44 patients), and death was directly due to mediastinitis. The patient was a 74-year-old man with El Oakley type IIIB mediastinitis. Prior cardiac surgery was a Bentall procedure. Mediastinal and blood cultures were positive for MRSA. The patient had signs of septic shock at the time of mediastinitis diagnosis, and he died of multiorgan failure 14 days after wound debridement.

The majority of patients $(43 / 44 ; 97.8 \%)$ were extubated in the operating room after debridement and transferred to the ward within 24 hours. ICU stay and prolonged mechanical ventilation (PMV) were required in 1 patient $(2.2 \%)$.

Life-threatening complications occurred in 1 patient who experienced major mediastinal bleeding caused by a left internal thoracic artery graft rupture 12 days after NPWT. The graft was sutured, and the patient underwent a successful left anterior descending coronary angioplasty.

The length of stay was $24 \pm 33$ days (range, 9-78 days). The time required to sterilize mediastinal cultures was 
TABLE 2. Univariate analyses for mediastinitis-related death

\begin{tabular}{|c|c|c|c|c|}
\hline Variable & $\boldsymbol{P}$ & OR & $\begin{array}{c}\text { Lower } \\
\text { CI limit }\end{array}$ & $\begin{array}{c}\text { Upper } \\
\text { CI limit }\end{array}$ \\
\hline Age & .246 & 1.056 & 0.949 & 1.174 \\
\hline Sex & .608 & 1.029 & 0.973 & 1.09 \\
\hline Diabetes & .422 & 1.038 & 0.964 & 1.118 \\
\hline Obesity & .139 & 1.077 & 0.931 & 1.245 \\
\hline COPD & .422 & 0.963 & 0.894 & 1.037 \\
\hline Creatinine $>2$ & .118 & 1.083 & 0.926 & 1.267 \\
\hline Dialysis & .784 & 0.976 & 0.932 & 1.024 \\
\hline Vasculopathy & .688 & 0.974 & 0.924 & 1.026 \\
\hline Transfusion & .536 & 1.032 & 0.97 & 1.099 \\
\hline Anemia & .536 & 1.032 & 0.97 & 1.099 \\
\hline BITA & .291 & 0.952 & 0.866 & 1.048 \\
\hline Other than CABG alone & .246 & 1.056 & 0.949 & 1.174 \\
\hline LOS & .749 & 0.975 & 0.928 & 1.025 \\
\hline ICU recovery & .091 & 1.333 & 0.757 & 2.348 \\
\hline Inotrope & .181 & 1.067 & 0.94 & 1.211 \\
\hline Organism & .312 & 1.048 & 0.956 & 1.148 \\
\hline Blood infection & .312 & 1.048 & 0.956 & 1.148 \\
\hline Septic shock & .0001 & NA* & $\mathrm{NA}^{*}$ & NA* \\
\hline APACHE II at diagnosis & .182 & 1.143 & 0.88 & 1.385 \\
\hline
\end{tabular}

APACHE, Acute Physiology and Chronic Health Evaluation; BITA, bilateral internal thoracic artery; $C A B G$, coronary artery bypass grafting; $C I$, confidence interval; $C O P D$, chronic obstructive pulmonary disease; $I C U$, intensive care unit; $L O S$, length of stay; $N A$, not applicable; $O R$, odds ratio. *Risk estimate cannot be performed because of the singularity of the event and nonoccurrence in the nonevent of the analyzed factor ( 2 empty cells in the contingency table). Variables were recorded at the time of mediastinitis diagnosis.

$20.3 \pm 35$ days (range, 6-72 days). Six patients required more than 60 days to sterilize the mediastinal cultures. The mean time from flap procedure to hospital discharge was 5 days.

Reintervention for recurrent wound infection occurred in 2 patients $(4.5 \%) 8$ and 12 days after pectoralis flap wound closure. Both patients had a superficial wound infection without sternal involvement. Dehiscence was localized on the distal third of the wound. Patients were readmitted to the operating room. After debridement of the presternal tissues, NPWT was restarted until wound closure. Both patients had chronic renal failure and were treated with dialysis. Univariate analysis showed septic shock to be the only independent risk factor $(P<.0001)$ for mediastinitisrelated death (Table 2), and dialysis was found to be the only independent factor $(P<.0001)$ for reintervention (Table 3).

\section{DISCUSSION}

Several strategies have been described to improve the outcome of PSM, but optimal management is still controversial. The open wound approach allows infection control by serial dressing changes, but it has high mortality rate, up to $45 \%$, and major complications related to the management of an open sternum limited the use of this strategy.

Since 1963, the closed approach has been the treatment of choice in many centers. ${ }^{18,19}$ The primary closed Redon drainage technique received a large consensus, improving the mediastinitis mortality rate of approximately $20 \%$ in the last decade series. ${ }^{20,21}$ Age, operations other than CABG alone, McCabe class 2/3, APACHE II score, prolonged mechanical ventilation, persistently positive bacteremia, and MRSA mediastinitis were identified as mortality risk factors with this approach. ${ }^{13,20}$

On the other hand, Molina and colleagues ${ }^{22}$ combined irrigation and suction with the primary closure technique and obtained excellent results. The success rate was $98 \%$, with no mortality. In contrast with these excellent results, there was an aggressive irrigation flow rate of approximately $400 \mathrm{~mL} / \mathrm{h}(9600 \mathrm{~mL} / \mathrm{d})$ for 7 days with delayed patient mobilization.

Douville and co-workers ${ }^{23}$ modified the traditional open dressing approach. They suggested that wound debridement and sternal rewiring followed by delayed closure of presternal tissues could be effective in mediastinitis treatment and reported a mortality rate of $14 \%$.

Since the introduction of VAC by Argenta and Morykwas in $1997,{ }^{10}$ the device has become a widely accepted option in the management of chronic and difficult wounds. During the last decade, several studies have reported the successful use of VAC therapy in mediastinitis treatment, ${ }^{24,25}$ and currently it is widely used as the first-line approach to this complication. The Lund University group ${ }^{26}$ reported excellent results with VAC therapy in terms of safety, failure rate, and survival compared with conventional treatment. Moreover, several effects on wound environment have been described. VAC therapy increases muscle blood flow ${ }^{27,28}$ and reduces fluid excess and bacterial colonization, which in turn promote granulation tissue and accelerate wound healing.

Despite the mortality rate improving from $50 \%$ to $10 \%$ to $15 \%$ according to recent studies, ${ }^{11-20}$ the outcome of PSM is still unpredictable. With the exception of urgent debridement and culture-guided antibiotic regimen, optimal treatment is still controversial.

This report analyzed a cohort of 44 consecutive patients with mediastinitis with a mediastinitis-related mortality of $2.2 \%$. Our protocol was based on 2 different stages. The first stage was characterized by urgent wound debridement and NPWT until wound sterilization, and the second stage was based on elective chest wall closure by pectoralis major muscle advancement flap.

Data analysis of our population showed a radical gain in patients' clinical status during the early phase of the first stage of treatment, changing the severity of the average clinical condition and in turn allowing a satisfactory recovery rate. Statistical analysis showed a significant improvement of APACHE II score 24 hours after wound debridement and NPWT application, independently of any other variables included in the study (Table 1). The only mediastinitisrelated death was in a patient in septic shock at the time of 
TABLE 3. Univariate analyses of need for reintervention

\begin{tabular}{llllr}
\hline \multicolumn{1}{c}{ Variable } & \multicolumn{1}{c}{$\boldsymbol{P}$} & OR & $\begin{array}{c}\text { Lower } \\
\text { CI limit }\end{array}$ & $\begin{array}{c}\text { Upper } \\
\text { CI limit }\end{array}$ \\
\hline Age & .118 & 0.88 & 0.761 & 1.017 \\
Sex & .363 & 1.094 & 0.988 & 1.211 \\
Diabetes & .155 & 1.125 & 0.985 & 1.285 \\
Obesity & .22 & 0.901 & 0.799 & 1.014 \\
COPD & .845 & 0.781 & 0.065 & 9.34 \\
Creatinine $>2$ & .144 & 5.455 & 0.449 & 66.31 \\
Dialysis & .00001 & NA* & NA* & NA* \\
Vasculopathy & .476 & 0.921 & 0.839 & 1.011 \\
Transfusion & .807 & 0.733 & 0.06 & 8.915 \\
Anemia & .807 & 0.733 & 0.6 & 8.915 \\
BITA & .496 & 0.432 & 0.036 & 5.145 \\
Other than CABG alone & .721 & 0.639 & 0.054 & 7.617 \\
ICU recovery & .57 & 0.925 & 0.847 & 1.01 \\
Inotrope & .91 & 0.867 & 0.072 & 10.382 \\
Organism & .55 & 0.476 & 0.04 & 5.671 \\
Blood infection & .55 & 0.476 & 0.04 & 5.671 \\
Septic shock & .784 & 0.93 & 0.857 & 1.011 \\
APACHE II at diagnosis & .398 & 0.917 & 0.831 & 1.012 \\
\hline APACHE, Acte Phy
\end{tabular}

APACHE, Acute Physiology and Chronic Health Evaluation; BITA, bilateral internal thoracic artery; $C A B G$, coronary artery bypass grafting; $C I$, confidence interval; $C O P D$, chronic obstructive pulmonary disease; $I C U$, intensive care unit; $N A$, not applicable; $O R$, odds ratio. *Risk estimate cannot be performed because of the singularity of the event and nonoccurrence in the nonevent of the analyzed factor ( 2 empty cells in the contingency table). Variables were recorded at the time of mediastinitis diagnosis.

mediastinitis diagnosis. As evidenced in the recent literature, age, type of previous cardiac surgery, persistently positive bacteremia, severity of illness, and cultures positive for MRSA were identified as mediastinitis mortality risk factors at the time of the diagnosis. ${ }^{13,14,21}$ Even if comparison with other studies might not be fully appropriate, in our series, none of these variables influenced the outcome, which is instead heavily conditioned by septic shock.

PMV after wound debridement has been identified as a strong mortality risk factor in patients with mediastinitis, with a 20 -fold increase in mortality rate. In a large series, showed that patients weaned from the ventilator within 3 days after surgery had a low mediastinitis mortality rate $(\sim 2 \%)$ regardless of the association with other mediastinitis mortality risk factors, highlighting the importance of this phase in the outcome of this complication. Extensive debridement and primary closure, as performed in the conventional closed technique, represent an aggressive approach that might per se contribute to PMV in such a critical population. On the contrary, NPWT minimizes surgical invasiveness at the time of debridement. Repeated dressing changes and continuous wound drainage, ensured by the vacuum, allow effective infection control and avoid extensive debridement and wide exposure of bleeding and viable tissues. Moreover, negative pressure applied to the sponge immediately restores an effective sternal stabilization, promoting the contribution of a stable sternum to the respiratory function without the need for more complex surgical solutions. In our series, except for 1 patient in septic shock at the time of mediastinitis diagnosis who required mechanical ventilation before debridement, no patients required mechanical ventilation or ICU stay for more than 12 hours after NPWT.

We used the APACHE II score because it efficiently acts as a single parametric value of the overall clinical status. The analysis of all values constituting the APACHE II score demonstrated that respiratory function, hemoglobin, and electrolytes were invariably normalized immediately after NPWT, showing how this treatment affects the clinical status of patients in a short time frame. This approach contributes to clinical recovery, promoting effects of medical therapy in terms of blood transfusion, hydroelectrolytic balance, mobilization, and so forth.

The time required to sterilize mediastinal cultures was $20.3 \pm 35$ days. During this period, complete mobilization and general parameters normalization were progressively achieved, allowing chest wall reconstruction with a more complex surgical approach in patients. Negative pressure wound closure without muscle flaps has been described in patients with compromised conditions or atrophied tissues who could not tolerate surgical closure. ${ }^{29,30} \mathrm{We}$ used this approach in patients with recurrence of superficial dehiscence after the flap procedure, who had a problematic re-closure as the result of severely atrophied presternal tissues.

In our series, wound closure was performed by elective sternal reapproximation and pectoralis muscle flap. At the time of culture sterilization, the wound appeared clean and granulating, but dead space was generally too large and presternal tissues were too stiff to be sutured in a standard fashion. A pectoralis muscle advancement flap was needed to fill the presternal dead space, minimizing tensions on the sutures. Sternal rewiring was feasible in all patients with 6 to 8 single interrupted wires. In the majority of patients, wires were positioned only in the anterior surface of the sternum to avoid further dissections or organs injury. Fibrotic tissue growth below the sternum significantly reduces sternal mobility, contributing to a stable osteosynthesis without the need for adjunctive devices.

\section{Study Limitations}

This is a retrospective study conducted in a relatively small sample of patients. However, we describe the whole experience in the treatment of mediastinitis at a single center over a 6-year period. Data were collected prospectively in a dedicated database, and the same surgeon performed all procedures. Before 2006, the approach to mediastinitis was based on open packing or closed drainage depending on the surgeon's preferences, and data were not collected prospectively. This aspect prevented us from performing a comparative study. 


\section{CONCLUSIONS}

A simple and light wound debridement with sternal stabilization by NPWT is fundamental for early postoperative mobilization. In a short time frame, this allows a significant improvement in clinical status as determined by the APACHE II score. Wound sterilization and complete clinical recovery were invariably achieved before surgical closure. In this setting, sternal rewiring and pectoralis muscle advancement flap has proven to be safe and effective with an optimal cosmetic result and a high success rate. With this approach, septic shock at the time of diagnosis has been identified as the only independent risk factor for mediastinitis-related mortality.

\section{References}

1. Francel TJ, Kouchoukos NT. A rational approach to wound difficulties after sternotomy: the problem. Ann Thorac Surg. 2001;72:1411-8.

2. Mossad SB, Serkey JM, Longworth DL, Cosgrove DM III, Gordon SM. Coagulase-negative staphylococcal sternal wound infections after open heart operations. Ann Thorac Surg. 1997;63:395-401.

3. Loop FD, Lytle BW, Cosgrove DM, Mahfood S, McHenry MC, Goormastic M, et al. Sternal wound complications after isolated coronary artery bypass grafting: early and late mortality, morbidity and cost of care. Ann Thorac Surg. 1990;49: 179-87.

4. Borger MA, Rao V, Weisel RD, Ivanov J, Cohen G, Scully HE, et al. Deep sternal wound infection: risk factors and outcomes. Ann Thorac Surg. 1998;65:1050-6.

5. Hultman CS, Culbertson JH, Jones GE, Losken A, Kumar AV, Carlson GW, et al Thoracic reconstruction with the omentum: indications, complications, and results. Ann Plast Surg. 2001;46:242-9.

6. Gardlund B, Bitkover CY, Vaage J. Postoperative mediastinitis in cardiac surgery-microbiology and pathogenesis. Eur J Cardiothorac Surg. 2002;21: 825-30.

7. De Feo M, De Santo LS, Romano G, Renzulli A, Della Corte A, Utili R, et al. Treatment of recurrent Staphylococcal mediastinitis: still a controversial issue. Ann Thorac Surg. 2003;75:538-42.

8. Sarr MG, Gott VL, Townsend TR. Mediastinal infection after cardiac surgery. Ann Thorac Surg. 1984;38:415-23.

9. Morykwas MJ, Argenta LC, Shelton-Brown EI, McGuirt W. Vacuum assisted closure: a new method for wound control and treatment: animal studies and basic foundation. Ann Plast Surg. 1997;38:553-62.

10. Argenta LC, Morykwas MJ. Vacuum assisted closure. A new method for wound control and treatment: clinical experience. Ann Plast Surg. 1997;38:563-77.

11. Baillot R, Cloutier D, Montalin L, Cote L, Lellouche F, Houde C, et al. Impact of deep sternal wound infection management with vacuum assisted closure therapy followed by sternal osteosynthesis: a fifteen-year review of 23,499 sternotomies. Eur J Cardiothorac Surg. 2010;37:880-7.
12. Vos RJ, Yilmaz A, Sonker U, Kelder JC, Kloppenburg TL. Vacuum-assisted closure of post-sternotomy mediastinitis as compared to open packing. Interact Cardiovasc Thorac Surg. 2012;14:17-21.

13. Trouillet JL, Vuagnat A, Combes A, Bors V, Chastre J, Gandjbakhch I, et al. Acute poststernotomy mediastinitis managed with debridement and closeddrainage aspiration: factors associated with death in the intensive care unit. $J$ Thorac Cardiovasc Surg. 2005;129:518-24.

14. De Feo M, Renzulli A, Ismeno G, Gregorio R, Della Corte A, Utili R, et al. Variables predicting adverse outcome in patients with deep sternal wound infection. Ann Thorac Surg. 2001;71:324-31.

15. Garner J, Jarvis WR, Emori GT, Horan TC, Hughes J. CDC definitions for nosocomial infections 1988. Am J Infect Control. 1988;16:128-40.

16. El Oakley R, Wright J. Post-operative mediastinitis: classification and management. Ann Thorac Surg. 1996;61:1036-46.

17. Knaus WA, Draper EA, Wagner DP, Zimmerman JE. APACHE II: a severity of disease classification system. Crit Care Med. 1985;13:818-29.

18. Shumacker HB, Mandelbaum I. Continuous antibiotic irrigation in the treatment of infection. Arch Surg. 1963;86:384-7.

19. Durandy Y, Batisse A, Bourel P, Dibie A, Lemoine G, Lecompte Y. Mediastinal infection after cardiac operation. A simple closed technique. J Thorac Cardiovasc Surg. 1989;97:282-5.

20. Kirsch M, Mekontso-Dessap A, Houel R, Giroud E, Hillion ML, Loisance DY. Closed drainage using redon catheters for poststernotomy mediastinitis: results and risk factors for adverse outcome. Ann Thorac Surg. 2001;71:1580-6.

21. Poncelet AJ, Lengele B, Delaere B, Zech F, Glineur D, Funken JC, et al. Algorithm for primary closure in sternal wound infection: a single institution 10year experience. Eur J Cardiothorac Surg. 2008;33:232-8.

22. Molina JE, Nelson EC, Smith RR. Treatment of post-operative sternal dehiscence with mediastinitis: twenty-four year of a single method. J Thorac Cardiovasc Surg. 2006;132:782-7.

23. Douville EC, Asaph JW, Dworkin RJ, Handy JR Jr, Canepa CS Grunkemeier GL, et al. Sternal preservation: a better way to treat most sternal wound complications after cardiac surgery. Ann Thorac Surg. 2004;78:1659-64.

24. Gustafsson RI, Sjögren J, Ingemansson R. Deep sternal wound infection: a sternal sparing technique with vacuum assisted closure therapy. Ann Thorac Surg. 2003;76:2048-53.

25. Song DH, Wu LC, Lohman RF, Gottlieb LJ, Franczyk M. Vacuum assisted closure for the treatment of sternal wounds: the bridge between debridement and definitive closure. Plast Reconstr Surg. 2003;111:92-7.

26. Sjögren J, Gustafsson R, Nilsson J, Malmsjö M, Ingemansson R. Clinical outcome after poststernotomy mediastinitis: vacuum-assisted closure versus conventional treatment. Ann Thorac Surg. 2005;79:2049-55.

27. Wackenfors A, Gustafsson R, Sjogren J, Algotsson L, Ingemansson R Malmsjo M. Blood flow responses in the peristernal thoracic wall during vacuum-assisted closure therapy. Ann Thorac Surg. 2005;79:1724-31.

28. Petzina R, Gustafsson L, Mokhtari A, Ingemansson R, Malmsjo M. Effect of vacuum-assisted closure on blood flow in the peristernal thoracic wall after internal mammary artery harvesting. Eur J Cardiothorac Surg. 2006;30:85-9.

29. Calcaterra D, Garcia-Covarrubias L, Ricci M, Salerno TA. Treatment of mediastinitis with wound-vacuum without muscle flaps. J Card Surg. 2009;24:512-4.

30. Oeltjen JC, Panos AL, Salerno TA, Ricci M. Complete vacuum-assisted sternal closure following neonatal cardiac surgery. J Card Surg. 2009;24:748-50. 